JOURNAL OF ETHNOBIOLOGY

AND ETHNOMEDICINE

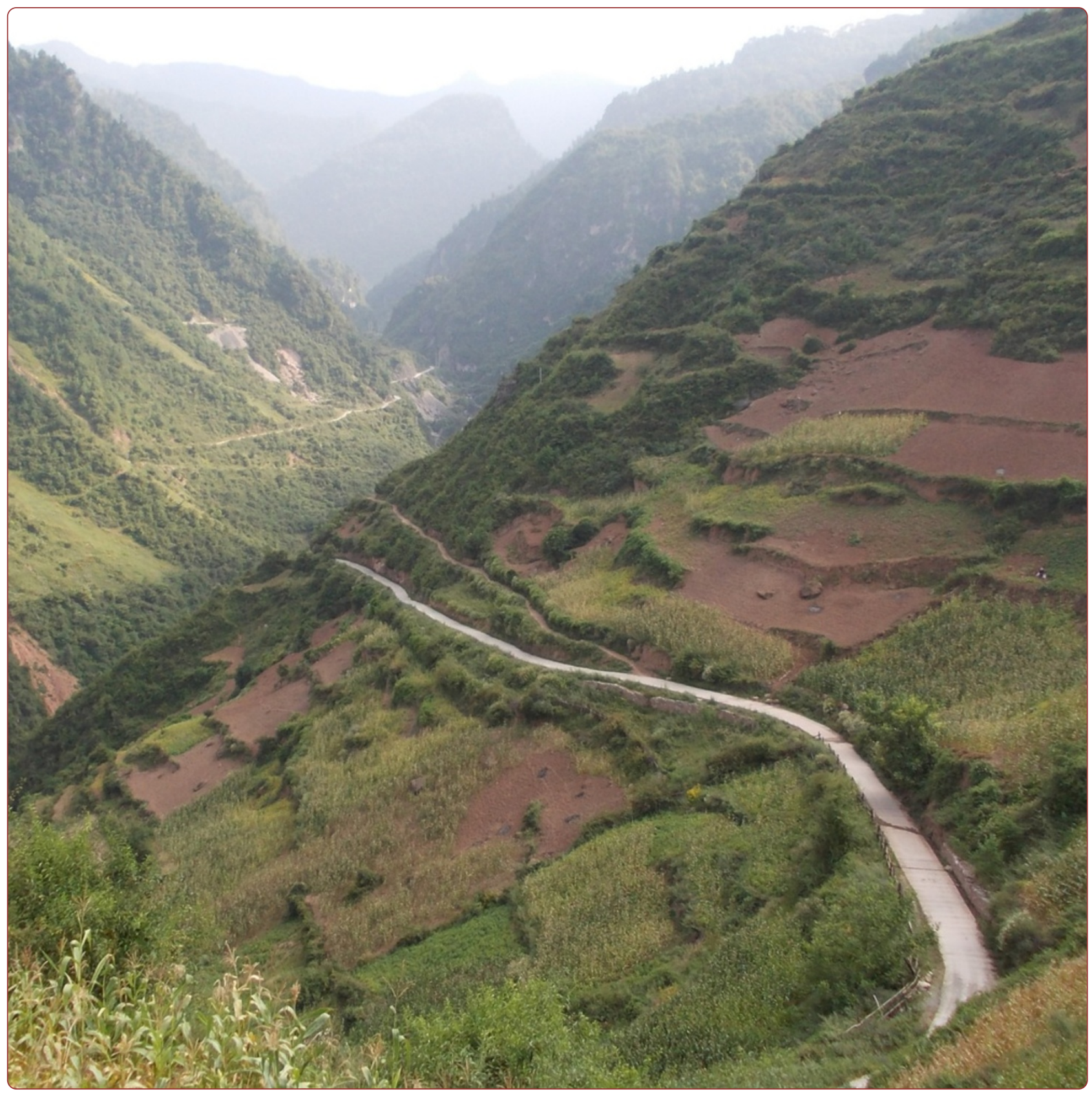

\title{
Wild food plants used by the Tibetans of Gongba Valley (Zhouqu county, Gansu, China)
}

Kang et al. 


\title{
Wild food plants used by the Tibetans of Gongba Valley (Zhouqu county, Gansu, China)
}

Yongxiang Kang ${ }^{1}$, Łukasz Łuczaj ${ }^{2 *}$, Jin Kang ${ }^{1}$, Fu Wang ${ }^{1}$, Jiaojiao Hou ${ }^{1}$ and Quanping Guo ${ }^{3}$

\begin{abstract}
Background: The ethnobotany of Tibetans is a seriously under-studied topic. The aim of the study was to investigate knowledge and use of wild food plants in a valley inhabited by Tibetans in the Gannan Tibetan Autonomous Region.

Methods: The field research was carried out in a wooded mountain valley in 9 neighbouring villages the Zhouqu (Brugchu) county, and comprised 17 interviews with single informants and 14 group interviews, involving 122 people altogether.

Results: We recorded the use of 81 species of vascular plants from 41 families. Fruits formed the largest category, with 42 species, larger than the wild greens category, with 36 species. We also recorded the culinary use of 5 species of edible flowers, 7 species with underground edible organs and 5 taxa of fungi. On average, 16.2 edible taxa were listed per interview (median - 16). Green vegetables formed the largest category of wild foods (mean - 8.7 species, median - 9 species), but fruits were listed nearly as frequently (mean - 6.9, median - 6). Other categories were rarely mentioned: flowers (mean - 0.2, median - 0), underground edible parts (mean - 0.3, median - 0) and mushrooms (mean - 1.5, - median 1). Wild vegetables are usually boiled and/or fried and served as side-dishes (cai). They are often lacto-fermented. Wild fruits are mainly collected by children and eaten raw, they are not stored for further use. The most widely used wild vegetables are: Eleuterococcus spp., Pteridium aquilinum, Helwingia japonica, Aralia chinensis, Allium victorialis, Pteridium aquilinum, Ixeris chinensis, Thlaspi arvense and Chenopodium album. The culinary use of Caltha palustris as a green vegetable is very interesting. In its raw state, marsh marigold is a toxic plant, due to the presence of protoanemonin. In this area it is dried or lactofermented before use. The most commonly eaten fruits are: Pyrus xerophila, Prunus salicina, Berchemia sinica, Rubus spp. and Eleagnus umbellata.
\end{abstract}

Conclusions: The number of wild taxa eaten in the studied valley is relatively large compared to most studies from around the world. However, compared to the northern slope of the Qinling, in Shaanxi, the list is considerably shorter, in spite of the similar methodology applied and similar research effort involved.

Keywords: Wild edible plants, Brugchu, Ethnobotany, Ethnomycology, Caltha palustris, Lactic fermentation, Lacto-fermented

\footnotetext{
* Correspondence: lukasz.luczaj@interia.pl

${ }^{2}$ Department of Botany and Biotechnology of Economic Plants, Institute of Applied Biotechnology and Basic Sciences, University of Rzeszów, Werynia 502, 36-100 Kolbuszowa, Poland

Full list of author information is available at the end of the article
} 


\section{Background}

Wild food plants still function as an important part of human nutrition in many parts of the globe. Although nowadays they usually provide only a minor proportion of daily calories, they are important sources of vitamins, microelements and other health supporting chemicals. They have always enabled human survival in times of crises [1-5]. The diversity of wild food plants consumed depends mainly on which species are available in sufficient quantity in the surrounding vegetation, the socioeconomic status of the population and cultural attitudes towards different categories of wild food. China is a very interesting arena for studies on wild food use traditions, as in all three above-mentioned aspects there are conditions that support the wide use of plants [6]. It contains an immense diversity of habitats and possesses numerous biodiversity hotspots. Some of its populations in remote rural areas still have a relatively low economic status, which binds people to traditional subsistence agriculture and exploitation of natural resources. Moreover, the rural population exploits different categories of wild food, including numerous species of wild vegetables, and is in itself diverse, consisting of many ethnic minorities, which differ in their plant utilization habits.

The ethnobotany research in China, concerning wild food plants, has so far been concentrated mainly on Yunnan [7-12] and Inner Mongolia [13,14], also as far as wild food is concerned. Although some papers on the use of wild food plants have been published from other parts of China (e.g. Sichuan [15,16], Hunan [17] etc.), some provinces are seriously under-studied ethnobotanically. An example of such a place is the province of Gansu, for which we found only one small article on wild vegetables, and only for the easternmost part of the province inhabited by Han Chinese [18]. Gansu is an ethnic mosaic of a few ethnic groups: Han Chinese, Hui and Tibetans [19]. In spite of the tremendous cultural diversity of different Tibetan ethnic groups and their diverse plant use, there is relatively little ethnobotanical literature devoted to the Tibetan people in China $[12,20-22]$. There is a particularly scarce ethnobotanical literature concerning the use of wild food plants by Tibetans, also in other provinces, with only one major article on the wild food uses of the Tibetans in Shangri-La (NW Yunnan) [12].

Although Tibetans are classified in China as one minority (藏族, Zangzu), they consist of a mosaic of several Tibetan languages, dialects and ways of life, with six main groups/dialects, out of which three occur in China classified as Ü-Tsang, Kham and Amdo [23]. The linguistic situation on the Gansu - Sichuan border is little studied, and it is one of the regions in which ever newer dialects are described (e.g. [24]). Some linguists distinguish over twenty separate distinct languages in the Tibetan group of languages [25]. As Nicolas Tournandre puts it: "there are 220 'Tibetan dialects' derived from

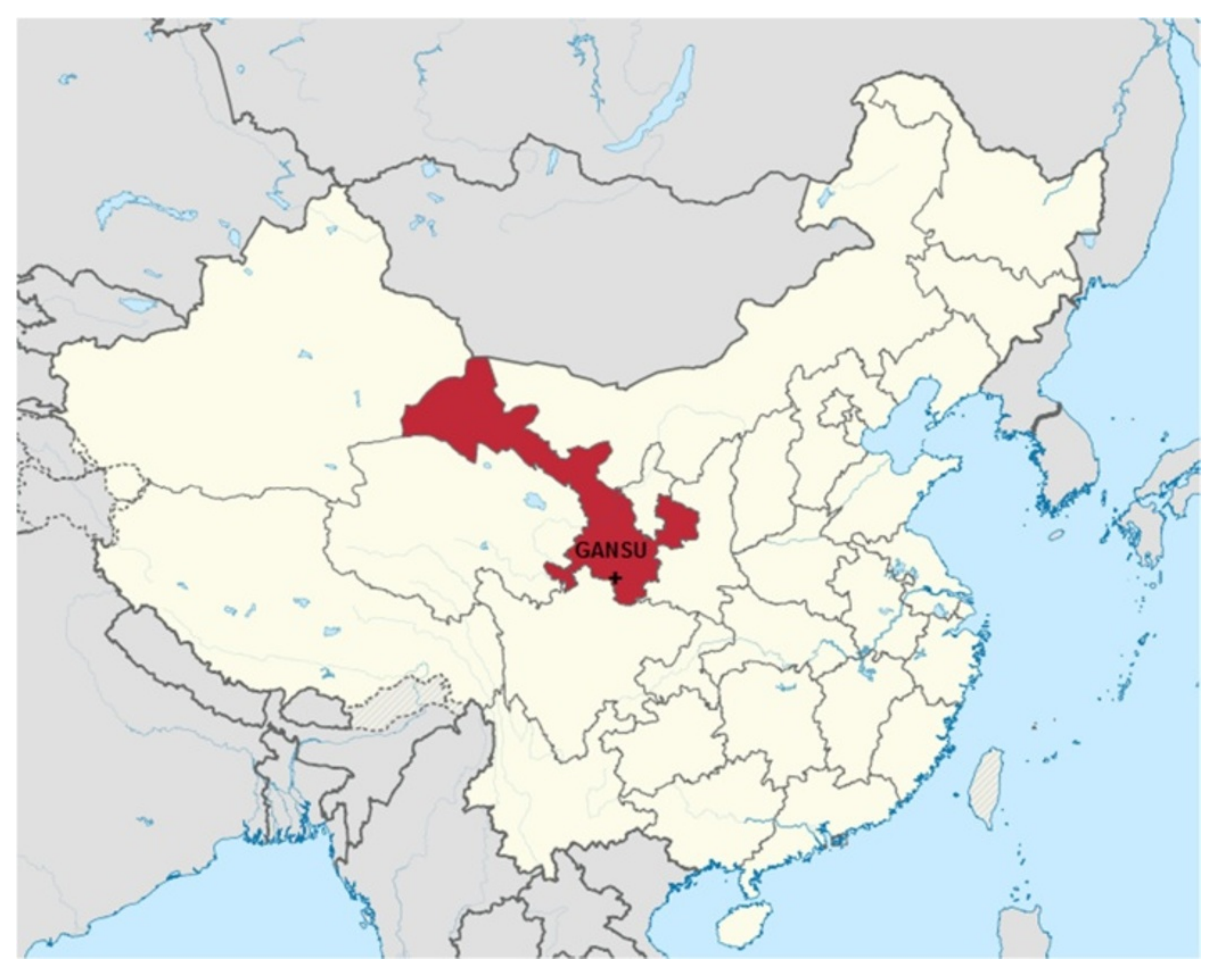

Figure 1 The location of the study area in China. 


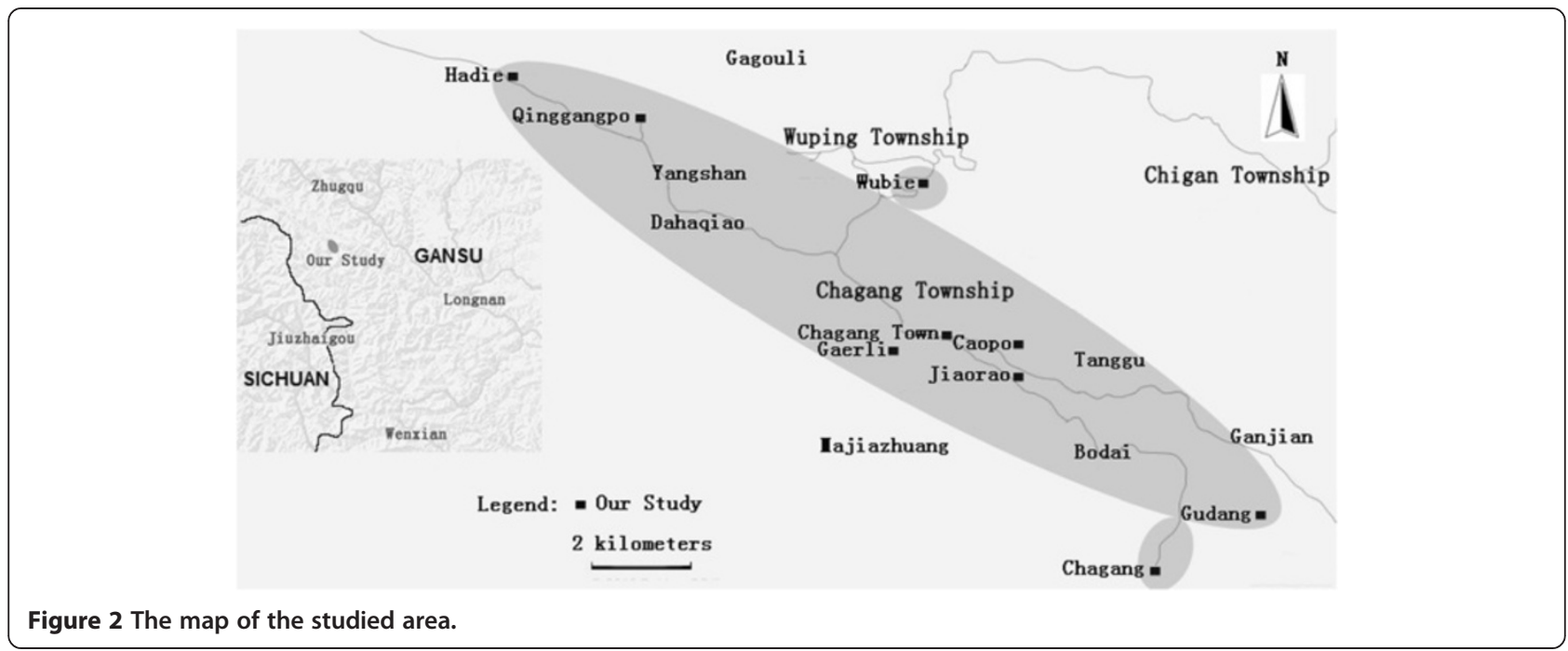

Old Tibetan and nowadays spread across 5 countries: China, India, Bhutan, Nepal and Pakistan which may be classed within 25 dialect groups, i.e. groups which do not allow mutual intelligibility. (...) Thus if we set aside the notion of standardization, I believe it would be more appropriate to speak of 25 languages derived from Old Tibetan. This is not only a terminological issue but it gives an entirely different perception of the range of variation. When we refer to 25 languages, we make clear that we are dealing with a family comparable in size to the Romance family which has 19 groups of dialects".

To fill the gap in the ethnobotanical exploration of the northwestern part of China we aimed at documenting the use of wild food plants in one Tibetan valley in SW Gansu, on the edge of the Qinling Mountains and the
Tibetan Plateau. As we have been, since 2011, carrying out similar research in other parts of the Qinling, inhabited by Han Chinese, one of the research questions also compared the pattern of wild food plant use with our previous studies [26-28].

\section{Methods}

\section{Study site}

The province of Gansu is located in northwest China, bordering Xinjiang and Qinhai in the west, Ningxia and Inner Mongolia in the north, Sichuan in the south and Shaanxi in the east (Figure 1). The vegetation changes from desert in the north and centre through dry grasslands to deciduous forests in the mountainous southern Gansu. In the south-west of Gansu, the Gannan Tibetan

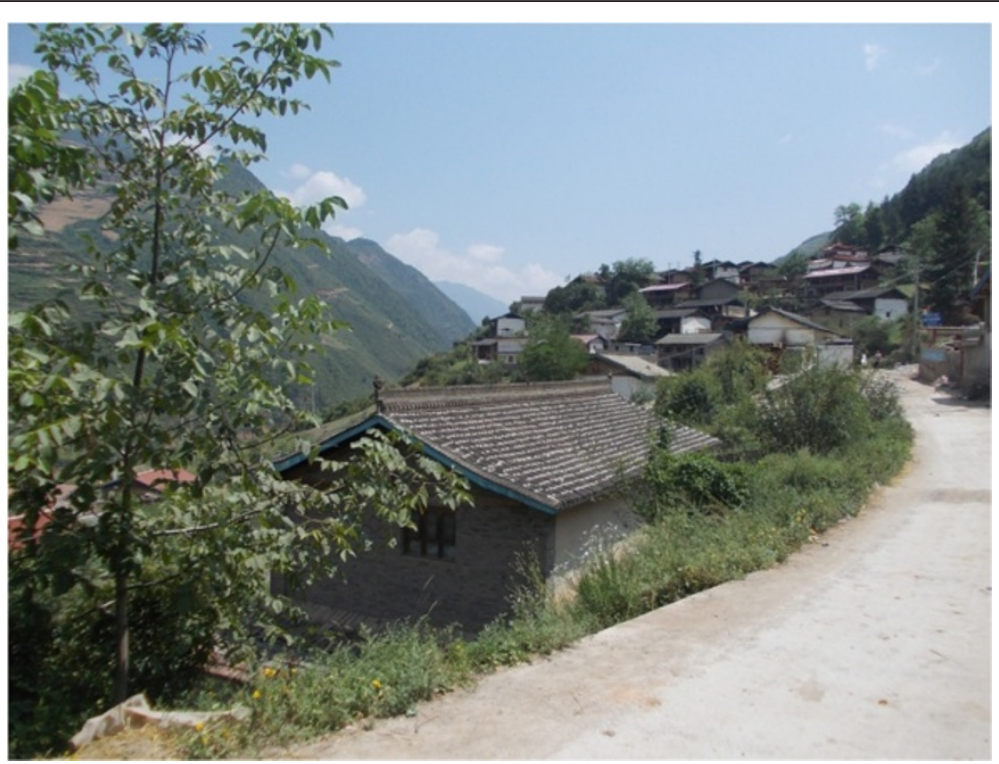

Figure 3 All the Tibetan villages in the valley are tight groups of homesteads located on hilltops. 


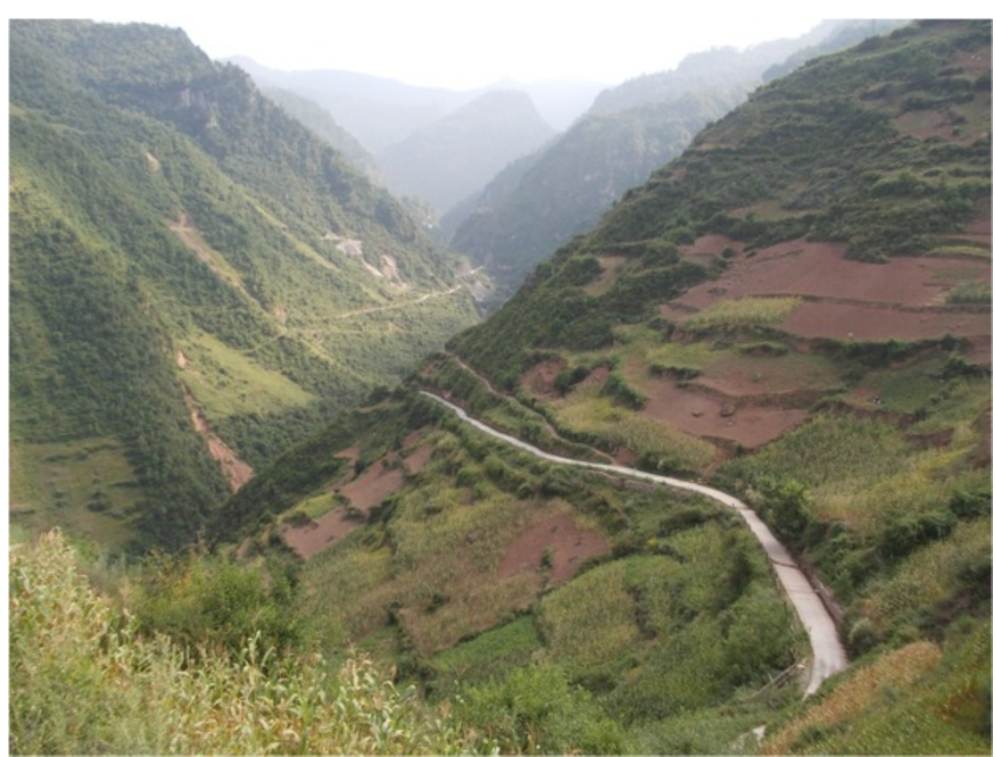

Figure 4 The landscape of Gongba valley around Chagang.

Autonomous Region was created where the Tibetan minority constitutes a large part of the population. Both the Chinese and Tibetans have a long history of settlement in this area as the region has formed a natural ethnic border between the Han Chinese and Tibetan speaking peoples [18].

Tibetans in Gannan constitute a very diverse collection of subsistence economies and speak a variety of dialects. In the northern part of the territory, in the grasslands, animal herders predominate. The Diebu (Tewo) Tibetans, although living among forests, live mainly from animal husbandry, whereas in the southern part of the region we studied, in some forested valleys, plant cultivation is the main source of subsistence.

The studied valley is located along a small river, Gongba, which is a tributary of Bailongjiiang (White Dragon River). Bailongjiang valley and its surrounding areas constitute a mountainous forefront of the Tibetan Plateau. We studied most Tibetan villages in two townships (镇 zhen): Chagang and Wuping [29]. The two townships are located in the southwestern part of the Zhouqu country (Brugchu or Zhugqu in Tibetan), beside the upper Gongba River basin,

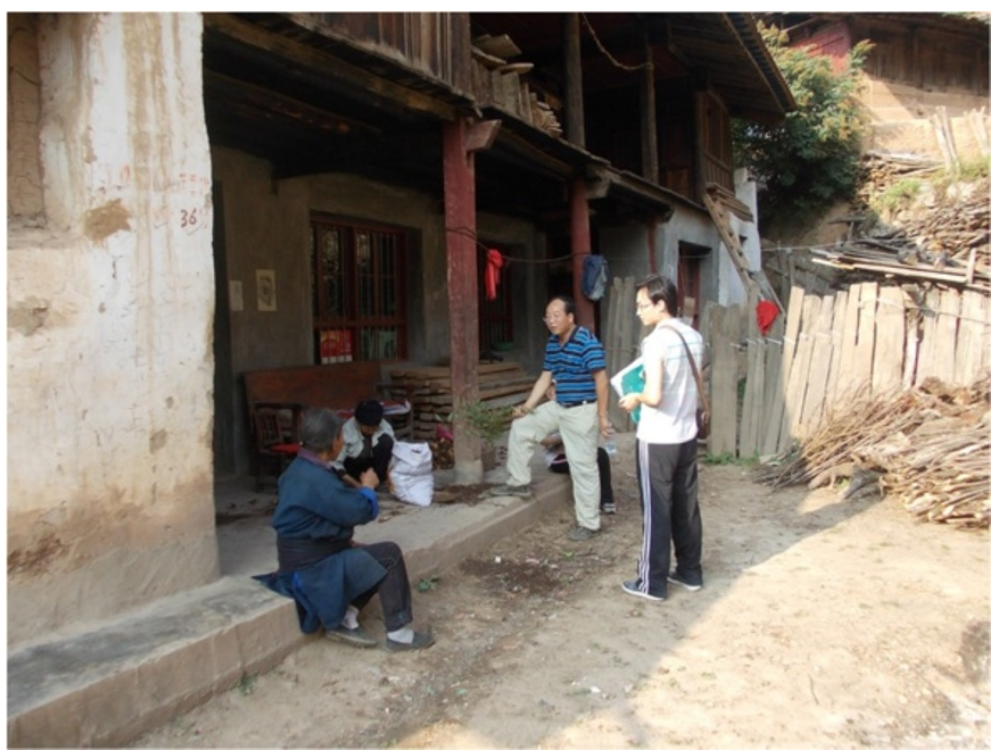

Figure 5 A typical two-storey house. 


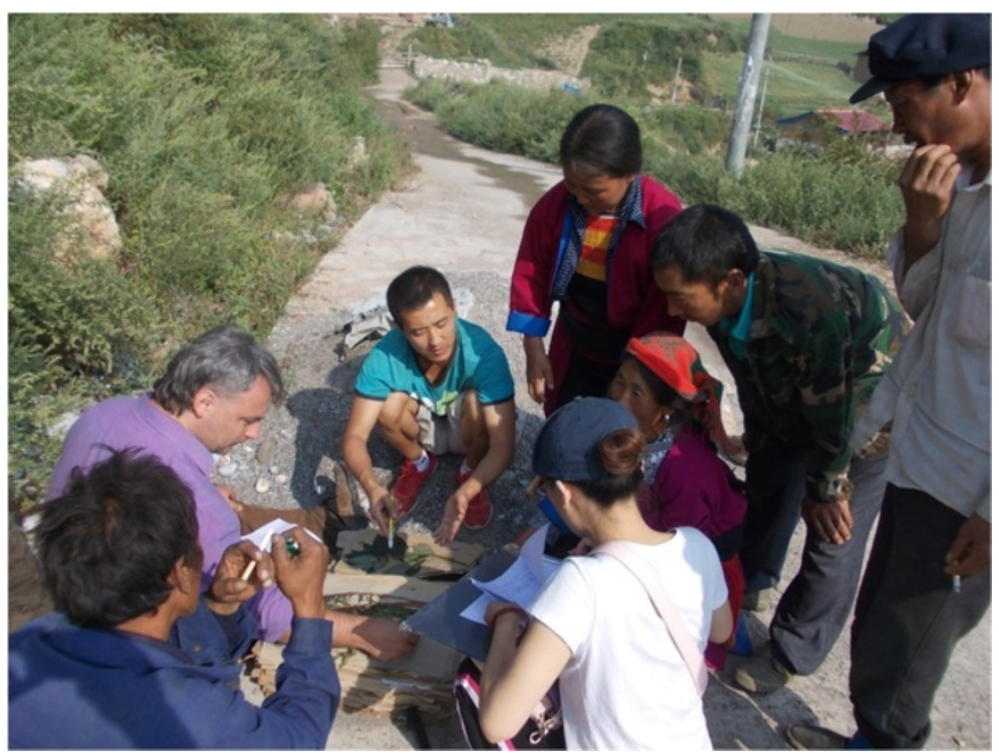

Figure 6 Local inhabitants eagerly took parts in interviews and specimen collection.

separated by massive mountain ranges both from the main part of Zhouqu country in the east and the Sichuan province in the southwest (Figures 2, 3 and 4).

The Chagang Township has an area of $157 \mathrm{~km}^{2}$. The average annual rainfall is $680 \mathrm{~mm}$ (mainly in summer months), the annual average temperature is $9.1^{\circ} \mathrm{C}$, and the frost-free period lasts 220 days. The average annual net income is 1376 Yuan. The total population is 3624, of which farmers account for 3197. There are Tibetan, Han and Hui in the township, but the Tibetan population accounts for $83 \%$ of the total population [29].

Wuping township is located upstream from the Chagang township. It has an area of $\left(429 \mathrm{~km}^{2}\right)$. The annual average temperature ranges between $6^{\circ} \mathrm{C}$ and $12^{\circ} \mathrm{C}$, the frost-free period lasts 160 to 200 days. It consists of 10 administrative villages, including 32 small settlements, with a total population of 6962. There are 6240 farmers, including 2889 Tibetans, who constitute $41.5 \%$ of the total population [29].

The studied villages are purely Tibetan and usually set at the top of a hill, with a few dozen houses, all very close to each other, e.g. Jiaorao Village, with 83 households and 483 inhabitants, Gudang - 62 houses, 300 people, Gaerli over 500 people and Hadie - 116 houses, 618 inhabitants [29]. We studied nine villages altogether, in Chagangzhen township (插岗镇): Jiaorao (角绕), Jiaoerli (噶儿里), Caopo (草坡), Qinggangpo (青岗坡), Chagang (插岗村), Gudang (古当), Wubie (吾别); in Wupingzhen township (武坪镇): Hadie (哈迭) and Wuping (武坪). The altitude ranged from 1782 to $2140 \mathrm{~m}$ a.s.l.

The studied population consists of subsistence farmers cultivating barley, potatoes, maize, flax, buckwheat, broad bean, pea, rape-seed and some fruit trees, e.g. apple, pear, walnuts and Sichuan pepper (Zanthoxylum bungeanum).
Table 1 Twenty most frequently mentioned wild food plants in the freelisting questionnaire

\begin{tabular}{lcc}
\hline Species & Part & $\begin{array}{c}\text { Frequency } \\
\text { (N = 31) }\end{array}$ \\
\hline $\begin{array}{l}\text { Eleuterococcus giraldii \& E. } \\
\text { leucorrhisus }\end{array}$ & Opening buds & 30 \\
Ferns in general ('zhala') & Frond stalks & 30 \\
Helwingia japonica & Young leaves & 29 \\
Aralia chinensis & Opening buds & 26 \\
Pyrus xerophila & Fruits & 20 \\
Prunus salicina & Fruits & 19 \\
Berchemia sinica & Fruits & 17 \\
Allium victorialis & Leaves & 16 \\
Pteridium aquilinum & Fronds & 16 \\
Rubus mesogaeus \& R. thibetanus & Fruits & 16 \\
Juglans cathayensis & Kernel & 14 \\
Thlaspi arvense & Young aerial parts & 14 \\
Eleagnus umbellata & Fruits & 13 \\
Ixeris chinensis & Young aerial parts, & 12 \\
Rubus amabilis & roots & 12 \\
Schisandra sphenanthera & Fruits & 12 \\
Fragaria pentaphylla & Fruits & 11 \\
Auricularia sp. & Fruits & 10 \\
Chenopodium album & Whole mushroom & 8 \\
Decaisnea fargesii & Foung aerial parts & 10 \\
Morchella spp. & Fruit pulp & 8 mushroom \\
Vitis piasezkii & & 8 \\
\hline & Fruits & 12 \\
\hline
\end{tabular}


Table 2 Wild food plants collected by at least two informants

\begin{tabular}{|c|c|c|c|c|c|}
\hline Family & Species & $\begin{array}{c}\text { Part } \\
\text { collected }\end{array}$ & $\begin{array}{l}\text { Local } \\
\text { Chinese } \\
\text { name }\end{array}$ & $\begin{array}{l}\text { Local Chinese } \\
\text { name (pinyin) }\end{array}$ & $\begin{array}{l}\text { Local Tibetan name (broad transcription of } \\
\text { pronunciation according to IPA) }\end{array}$ \\
\hline Actinidiaceae & Actinidia tetramera Maxim. & $\mathrm{F}$ & - & - & $\widehat{\text { dzitmədzile }}$ \\
\hline \multirow[t]{3}{*}{ Amaranthaceae } & Amaranthus retroflexus $L .{ }^{*}$ & L & 绿苋 & Liu Xian & Gir \\
\hline & Chenopodium album $\mathrm{L}$. & L & $\begin{array}{l}\text { 灰灰菜, 灰 } \\
\text { 条 }\end{array}$ & $\begin{array}{l}\text { Hui Hui Cai, Hui } \\
\text { Tiao }\end{array}$ & (ng)tieni \\
\hline & $\begin{array}{c}\text { Acroglochin persicarioides } \\
\text { Moq.* }\end{array}$ & L & 复灰条 & Fu Hui Tiao & tieniliəmə [literally 'sheep Chenopodium'] \\
\hline \multirow[t]{2}{*}{ Amaryllidaceae } & Allium victorialis $\mathrm{L}$. & L & 鹿角菜 & Luo Er Jiu & ze:/zit: \\
\hline & Allium sp. & $L$ & $\begin{array}{l}\text { 老葱, 野 } \\
\text { 蒜, 野煼 }\end{array}$ & $\begin{array}{l}\text { Lao Cong, Ye } \\
\text { Suan, Ye Cong }\end{array}$ & dzuoma, guo \\
\hline Anacardiaceae & Rhus verniciflua Stokes & L & 刺菜 & Ci Cai & sihei \\
\hline Apiaceae & Carum carvi $\mathrm{L}$. & $F(S P)$ & 野茴香 & Ye Hui Xiang & gini \\
\hline \multirow[t]{3}{*}{ Araliaceae } & $\begin{array}{l}\text { Eleutherococcus giraldii } \\
\text { (Harms) T.Nakai, }\end{array}$ & L & 五爪菜 & Wu Zhua Cai & si:zana, striana \\
\hline & $\begin{array}{c}\text { Eleutherococcus leucorrhizus } \\
\text { Oliv. }\end{array}$ & L & 五爪菜 & Wu Zhua Cai & si:zana, striana \\
\hline & Aralia chinensis $\mathrm{L}$. & L & 木兰头 & Mu lan Tou & liæ(n)mə, liəmu \\
\hline \multirow[t]{7}{*}{ Asteraceae } & Artemisia subdigitata Mattf.* & L & 牛尾蒿 & Niu Wei Hao & k(h)ieba, k(h)aba \\
\hline & Ixeris chinensis Nakai. & $L, R$ & 苦麻菜 & Ku Ma Cai & kuga, kuhua \\
\hline & $\begin{array}{c}\text { Prenanthes macrophylla } \\
\text { Franch.* }\end{array}$ & L & 山尖子 & Shan Jian Zi & pahu:, payu: \\
\hline & Saussurea sp. & L & 筒筒菜 & Tong Tong Cai & dżini \\
\hline & $\begin{array}{c}\text { Sinacalia tangutica (Maxim.) } \\
\text { B.Nord.** }\end{array}$ & R & 毛鞋 & Mao Hai & paibo, piaba, paibie \\
\hline & Sonchus oleraceus L. & L & 筒筒菜 & Tong Tong Cai & wu: [mainly], (ng)wu:, muwawu:, muwawu: \\
\hline & $\begin{array}{c}\text { Taraxacum cf mongolicum } \\
\text { Han.-Mzt }\end{array}$ & $L, R$ & 蒲公英 & Pu Gong Ying & aukuyua, aukəуa \\
\hline Athyriaceae & Athyriopsis sp. & L & 白彍 & Bai Jue & dzala \\
\hline \multirow[t]{3}{*}{ Brassicaceae } & Brassica campestris L. & L & 油菜 & You Cai & gu, gou \\
\hline & Cardamine macrophylla Willd. & L & 湿根菜 & Shi Gen Cai & tढ़idi \\
\hline & Thlaspi arvense L. & L & 苦根菜 & Ku Gen Cai & $\widehat{d z} a:(n)$ \\
\hline Campanulaceae & Adenophora stricta Miq. & $L, R$ & 泡参 & Pao Shen & $\widehat{d z} \partial$ mudżola \\
\hline \multirow[t]{6}{*}{ Caprifoliaceae } & Lonicera standishii Carr. & $\mathrm{F}$ & 养皮子 & Qiao Pi Zi & (k)alaiala, asa, kagudžie, kai/gai, ciese(ng) \\
\hline & $\begin{array}{l}\text { Lonicera tragophylla Hemsl. } \\
\text { ex Forb. \& Hemsl. }\end{array}$ & $\mathrm{Fl}$ & 鱼鱼花 & Yu Yu Hua & mudesibe, nimenu, dztmutza: \\
\hline & $\begin{array}{l}\text { Triosteum pinnatifidum } \\
\text { Maxim. }\end{array}$ & $\mathrm{F}$ & 白蛋 & Bai Dan & kelu, keiü, kaloilo, alaiala \\
\hline & Triosteum himalayanum Wall. & $\mathrm{F}$ & - & - & dzitben, $\widehat{d z} i b(i) e n$, dżisxxxniuga, $\widehat{d z i m a s i, ~ b o d z ̇ i m a ~}$ \\
\hline & Viburnum betulifolium Batalin & $\mathrm{F}$ & - & - & 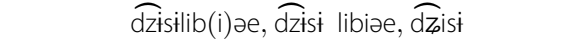 \\
\hline & $\begin{array}{l}\text { Viburnum glomeratum } \\
\text { Maxim. }\end{array}$ & $\mathrm{F}$ & - & - & \\
\hline Cephalotaxaceae & $\begin{array}{c}\text { Cephalotaxus sinensis (Rehder } \\
\text { \& E.H.Wilson) H.L.Li }\end{array}$ & $\mathrm{F}$ & 水柏子 & Shui Bai Zi & 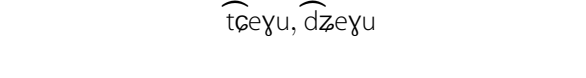 \\
\hline Cornaceae & Cornus japonica Thunb. & $\mathrm{F}$ & 荔枝 & Li Zhi & \\
\hline \multirow[t]{3}{*}{ Corylaceae } & Corylus sieboldiana Blume & $\mathrm{F}$ & 榛子 & Zhen Zi & 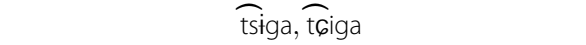 \\
\hline & $\begin{array}{c}\text { Corylus heterophylla Fisch. ex } \\
\text { Besser }\end{array}$ & $\mathrm{F}$ & 榛子 & Zhen Zi & 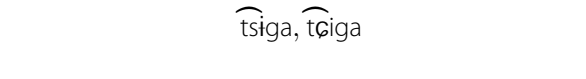 \\
\hline & Corylus ferox Wall. & $\mathrm{F}$ & 榛子 & Zhen Zi & 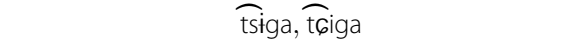 \\
\hline
\end{tabular}


Table 2 Wild food plants collected by at least two informants (Continued)

\begin{tabular}{|c|c|c|c|c|c|}
\hline Dennstaedtiaceae & $\begin{array}{c}\text { Pteridium aquilinum (L.) Kuhn } \\
\text { var. latiusculum }\end{array}$ & L & 阳蕨 & Yang Jue & 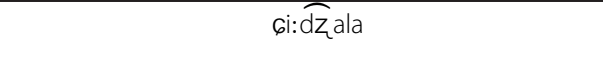 \\
\hline \multirow[t]{2}{*}{ Eleagnaceae } & Elaeagnus umbellata Thunb. & $\mathrm{F}$ & 尖子 & Jian Zi & hələ, șilə \\
\hline & Hippophae rhamnoides L. & $\mathrm{F}$ & & & laitsima, $\widehat{d z}$ imidzze, laitsiməgu, laigə \\
\hline Fabaceae & Sophora alopecuroides L. & $\mathrm{S}(\mathrm{SP})$ & 苦豆 & Ku Dou & not recorded \\
\hline Helwingiaceae & Helwingia japonica (Thunb.) F. & L & 叶里开花 & Ye Li Kai Hua & susąi \\
\hline Juglandaceae & Juglans cathayensis Dode & $F, F L$ & 野核桃 & Ye He Tao & $\begin{array}{c}\text { nina, dienanu, dienane }=\text { walnut flowers }[\text { die }=\text { walnut } \\
\text { tree }]\end{array}$ \\
\hline \multirow[t]{3}{*}{ Lamiaceae } & Caryopteris divaricata Maxim. & L & - & - & nina \\
\hline & Mentha haplocalyx Briq.* & L & 薄荷 & Bo He & Giucə, ઢiəzə \\
\hline & Stachys affinis Bunge & $\mathrm{R}$ & - & - & bazit \\
\hline \multirow[t]{2}{*}{ Lardizabalaceae } & Decaisnea fargesii Franch & $\mathrm{F}$ & 鬼指头 & Gui Zhi Tou & 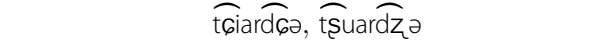 \\
\hline & $\begin{array}{l}\text { Akebia trifoliata (Thunb.) } \\
\text { Koidz. }\end{array}$ & $\mathrm{F}$ & - & - & dzəomedziıu \\
\hline Liliaceae & Lilium sp. & R & 大花, 百合 & Da Hua, Bai He & Giebu \\
\hline Malvaceae & Malva verticillata $L$. & L & 锦葵 & Jin Kui & (ng)nagu, ninagu, tamuda \\
\hline Meliaceae & $\begin{array}{l}\text { Toona sinensis (A.Juss.) M. } \\
\text { Roem. }\end{array}$ & L & 香椿 & Xiang Chun & tsaizime \\
\hline Moraceae & Morus australis Poir. & $\mathrm{F}$ & $\begin{array}{l}\text { 野桑子, 野 } \\
\text { 桑甚, }\end{array}$ & $\begin{array}{l}\text { Ye Sang Zi, Ye } \\
\text { Sang Shen }\end{array}$ & dusibielu, anazina \\
\hline \multirow[t]{2}{*}{ Onocleaceae } & Matteucia intermedia C.Chr. & L & 白蕨 & Bai Jue & dzala \\
\hline & $\begin{array}{l}\text { Matteucia struthiopteris (L.) } \\
\text { Tod. }\end{array}$ & L & 白蕨 & Bai Jue & džala \\
\hline Pinaceae & Pinus armandii Franch. & $\mathrm{S}$ & $\begin{array}{c}\text { 黄松, 苏木 } \\
\text { 头 }\end{array}$ & $\begin{array}{l}\text { Huang Song, Su } \\
\text { Mu Tou }\end{array}$ & tom $(u) g u, \operatorname{tom}(u) g \partial$ tor(ə), toż \\
\hline Plantaginaceae & Plantago depressa Willd.* & L & 车前 & Che Qian & 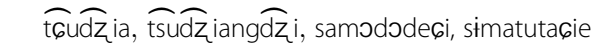 \\
\hline Polygonaceae & $\begin{array}{c}\text { Persicaria alata (Buch.Ham.) } \\
\text { Nakai* }\end{array}$ & L & 鬼菾 & Gui Qiao & khieredžoulemə, tuanledžou, lianmen \\
\hline Ranunculaceae & Caltha palustris $\mathrm{L}$. & L & 黄花菜 & Huang Hua Cai & huamien( (tsai), abububa \\
\hline \multirow[t]{2}{*}{ Rhamnaceae } & Berchemia sinica C.K.Schneid. & $\mathrm{F}$ & 提格儿 & Ti Ge Er & saü, sawi, saiu \\
\hline & $\begin{array}{c}\text { Rhamnus rosthornii E.Pritz. ex } \\
\text { Diels }\end{array}$ & $\mathrm{F}$ & - & - & 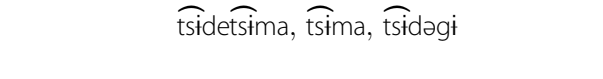 \\
\hline \multirow[t]{14}{*}{ Rosaceae } & Crataegus wilsonii Sarg. & $\mathrm{F}$ & 面梨 & Mian Li & da:da: \\
\hline & $\begin{array}{l}\text { Crataegus kansuensis E.H. } \\
\text { Wilson }\end{array}$ & $\mathrm{F}$ & 面梨 & Mian Li & da:da: \\
\hline & Fragaria pentaphylla Losinsk. & $\mathrm{F}$ & 漂子 & Piaozi & 6ü \\
\hline & Maddenia hypoleuca Koehne & $\mathrm{F}$ & - & - & Giana \\
\hline & Malus baccata (L.) Borkh. & $\mathrm{F}$ & 山定子 & Shan Ding Zi & səmənia \\
\hline & Neillia sinensis Oliv. & L & 茶格 & Cha Ge & mahadəzə, wahadəzə, wahadər, mahu৩tu \\
\hline & Prunus davidiana Franch. & $\mathrm{F}$ & 野毛桃 & Ye Mao Tao & ti:, ting, kuazə, kuebu, kuozuo, kuumo \\
\hline & Prunus salicina Lindl & $\mathrm{F}$ & $\begin{array}{l}\text { 苦李子, 野 } \\
\text { 李子 }\end{array}$ & Ku Li Zi, Ye Li Zi & dżile, đzilə \\
\hline & Prunus tomentosa Thunb. & $\mathrm{F}$ & - & - & nest \\
\hline & Pyrus xerophila T.T.Yu & $\mathrm{F}$ & 野酸梨 & Ye Suan Li & 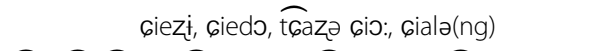 \\
\hline & Rosa brunonii Lindl. & $\mathrm{F}$ & 刺格 & $\mathrm{Ci} \mathrm{Ge}$ & 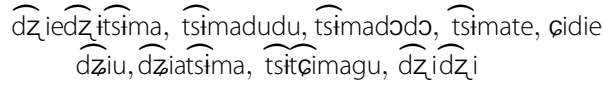 \\
\hline & Rubus amabilis Focke & $\mathrm{F}$ & 红帽子 & Hong Mao Zi & 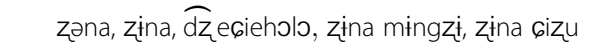 \\
\hline & Rubus mesogaeus Focke & $\mathrm{F}$ & 黑帽子 & Hei Mao Zi & zəona, żina \\
\hline & Rubus thibetanus Franch & $\mathrm{F}$ & 黑帽子 & Hei Mao Zi & zənna, zina, $\widehat{d z} u ə r$, duheni, ciehele, wunihebie \\
\hline
\end{tabular}


Table 2 Wild food plants collected by at least two informants (Continued)

\begin{tabular}{|c|c|c|c|c|c|}
\hline & $\begin{array}{c}\text { Rubus xanthocarpus Bureau \& } \\
\text { Franch. }\end{array}$ & $\mathrm{F}$ & 黄帽子 & Huang Mao Zi & 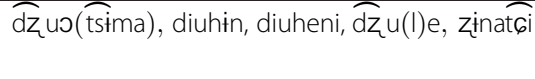 \\
\hline & Sorbus xanthoneura Rehder & $\mathrm{F}$ & 黄脉花楸 & $\begin{array}{l}\text { Huang Mai Hua } \\
\text { Qiu }\end{array}$ & duosi(Gieduo), dzuumą̧ias, džumacieda \\
\hline Rutaceae & $\begin{array}{c}\text { Zanthoxylum bungeanum } \\
\text { Maxim. }\end{array}$ & $L, F$ & 椒芽, 花椒 & Jiao Ya, Hua Jiao & iime \\
\hline \multirow[t]{2}{*}{ Salicaceae } & Populus davidiana Dode & $\mathrm{FL}$ & 杨树花 & Yang Shu Hua & - \\
\hline & Salix spp. & $\mathrm{FL}$ & 柳花 & Liu Hua & tanilo \\
\hline Santalaceae & Buckleya henryi Diels & $\mathrm{F}$ & - & & dżəma \\
\hline Saxifragaceae & Rodgersia aesculifolia Batalin** & $\mathrm{R}$ & 鬼灯擎 & Gui Deng Qing & hamahazi \\
\hline Schisandraceae & $\begin{array}{l}\text { Schisandra sphenanthera } \\
\text { Rehder \& E.H.Wilson }\end{array}$ & $\mathrm{F}$ & 五味子 & Wu Wei Zi & wi:, wəi \\
\hline Staphyleaceae & Staphylea holocarpa Hemsl. & L & 亮子尖 & Liang Zi Jian & 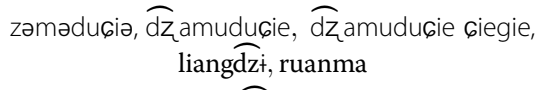 \\
\hline Thelypteridaceae & $\begin{array}{c}\text { Pseudocyclosorus } \\
\text { subochthoides (Ching) Ching }\end{array}$ & L & 白蕨 & Bai Jue & džala \\
\hline Urticaceae & Urtica fissa E.Pritz. ex Diels* & L & 菷麻 & Qian Ma & sana [mainly], sapadza [rarely] \\
\hline Vitaceae & Vitis piasezkii Maxim. & $\mathrm{F}$ & 野葡萄 & Ye Pu Tao & 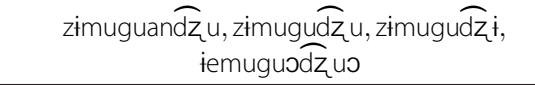 \\
\hline
\end{tabular}

Plant parts: L - young leaves, buds or other aerial parts; F - fruits; FL - inflorescences; R - roots, rhizomes etc.; S - seeds; SP - used as spice.

*controversial food plants, nowadays used by very few informants; regarded by most people as inedible or unattractive but usually widely used as food for pigs. **used only as famine food in 1959-61.

sthe accent always falls on the last syllable of the word.

More rarely fungi (mainly Auricularia and Grifola umbellata) and medicinal herbs (Gastrodia, Bupleurum chinense, Codonopsis) are cultivated.

\section{Data collection}

The field research was conducted in August 2013 using the Rapid Rural Appraisal approach [30,31], and included 31 freelisting interviews (17 interviews with single informants and 14 group interviews), which altogether involved 122 people (70 men and 52 women). The mean age of the participants was 50 (median 48, aged from 12 to 85; 52 women and 70 men). For the seventeen people (11 men and 6 women) who were interviewed separately the mean age was 53 , median 51 , youngest interviewee -23 , the oldest one -75$)$.

The listed taxa were identified using transect walks for cross-checking of the gathered herbarium specimens and semi-structured interviews with key informants (Figures 5 and 6). During freelisting we separately asked which species of wild vegetables (including underground organs), wild fruits and wild mushrooms were used. Making three separate freelists enabled the comparison of the use of these categories and helped elicit answers from the respondents, who categorized the studied wild products in the same way $[32,33]$. Freelists were made orally and written down on the spot by our team. The interviews were additionally recorded using a digital sound recorder. The interviews were carried out in standard Mandarin Chinese (most of the population is bilingual and attends a Chinese language school). We recorded the local Chinese language names as well as local Tibetan names and transcribed them according to the International Phonetic Alphabet.

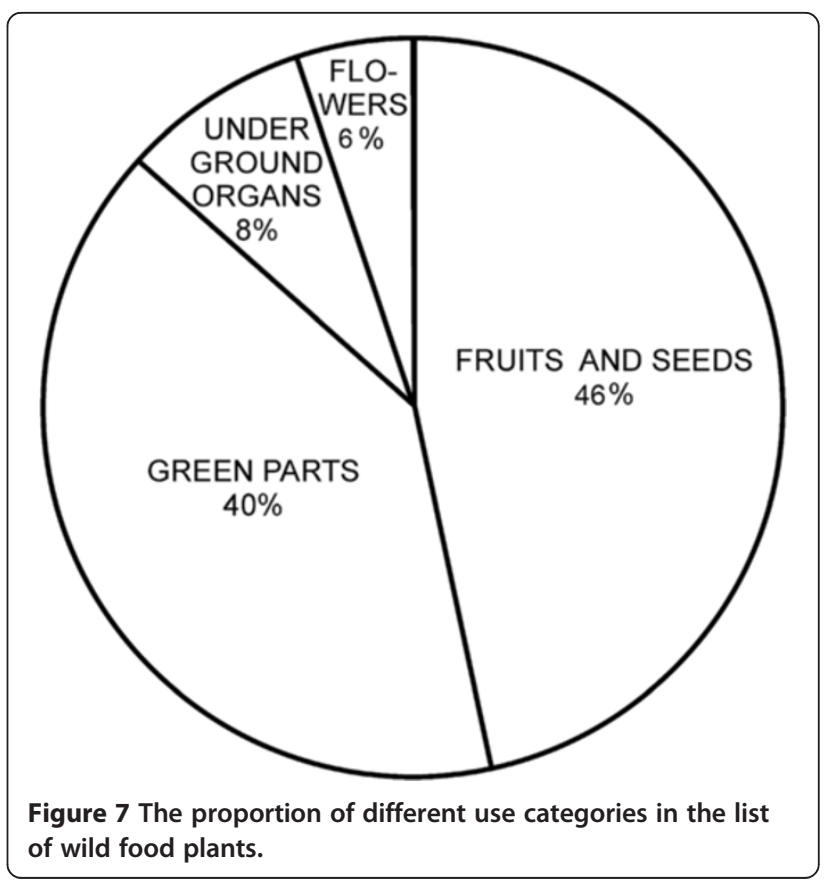


Table 3 Wild mushrooms collected for food mentioned by at least two informants

\begin{tabular}{|c|c|c|c|}
\hline Species & $\begin{array}{l}\text { Local } \\
\text { Chinese } \\
\text { name }\end{array}$ & $\begin{array}{l}\text { Local } \\
\text { Chinese } \\
\text { name } \\
\text { (pinyin) }\end{array}$ & $\begin{array}{l}\text { Local Tibetan name } \\
\text { (pronunciation } \\
\text { according to IPA) }\end{array}$ \\
\hline $\begin{array}{l}\text { Grifola umbellata } \\
\text { (Pers.) Pilát }\end{array}$ & 猪苓花 & $\begin{array}{l}\text { Zhu Ling } \\
\text { Hua }\end{array}$ & $\begin{array}{c}\text { dzuulinmo, } \overparen{d z} \text { ulinghua, } \\
\overparen{d z} \text { ulinghəmə }\end{array}$ \\
\hline $\begin{array}{l}\text { Morchella spp. } \\
\text { (mainly M. } \\
\text { angusticeps Peck) }\end{array}$ & 羊肚菌 & $\begin{array}{l}\text { Yang Du } \\
\text { Jun }\end{array}$ & 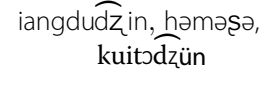 \\
\hline $\begin{array}{l}\text { Auricularia spp. } \\
\text { (mainly A. auricula- } \\
\text { judae (Bull.) Quél.) }\end{array}$ & 野木耳 & Ye Mu Er & ainou \\
\hline $\begin{array}{l}\text { "a red capped } \\
\text { mushroom" }\end{array}$ & 红蘑菇 & $\begin{array}{l}\text { Hong Mo } \\
\text { Gu }\end{array}$ & həmə, həməhə \\
\hline
\end{tabular}

The study started from a few recommended informants, including village leaders. These people were well-known to one of the authors of the article (Quanping Guo), who is the local representative of the forestry authorities and has spent much time with local residents working in the forests for 31 years. He also authored an article on local dible mushroom resources [34]. The rest of the interviewees were found by systematic walks through the village and surrounding fields, visiting houses and asking the inhabitants if they wanted to take part in the study. Voucher specimens are stored in the Department of Forestry, Northwest A\&F University in Yangling.

The research was carried out following the code of ethics of the American Anthropological Association [35] and the International Society of Ethnobiology Code of Ethics [36]. Oral prior informed consent was acquired. Statistics were calculated using the open access statistical package PAST $[37,38]$.

\section{Results and discussion}

We recorded the use of 81 species of vascular plants from 41 families (Tables 1 and 2; Figure 7). The Rosaceae family was the most represented. Fruits formed the largest category, with 41 species, larger than wild greens with 36 species. We recorded the culinary use of only five species of edible flowers and seven species with underground edible organs. The use of only five fungi taxa was recorded (Table 3). Most respondents knew both the Tibetan and Chinese names.

On average, 16.2 edible taxa were listed per interview (median - 16). The most listed category of wild foods was green vegetables (mean - 8.7 species, median - 9 species), but fruits were listed nearly as frequently (mean - 6.9, median - 6). Other category lists were very short: flowers (mean -0.2 , median -0$)$, underground edible parts (mean 0.3 , median - 0) and mushrooms (mean - 1.5, - median 1).

In group interviews we obtained only slightly more species per list (e.g. fruits - 6.4 species in individual ones, 6.9 in group ones; green vegetables -8.3 and 9.2 respectively). The difference between individual and group interviews is small, probably due to the fact that some of the best informants were interviewed separately.

Wild vegetables are usually boiled and/or fried and served as side-dishes (cai). Wild fruits are collected mainly by children and eaten raw, they are not stored for further use. Mushrooms are usually fried. During the 1959-61 famine Sinacalia tangutica tubers (one of the

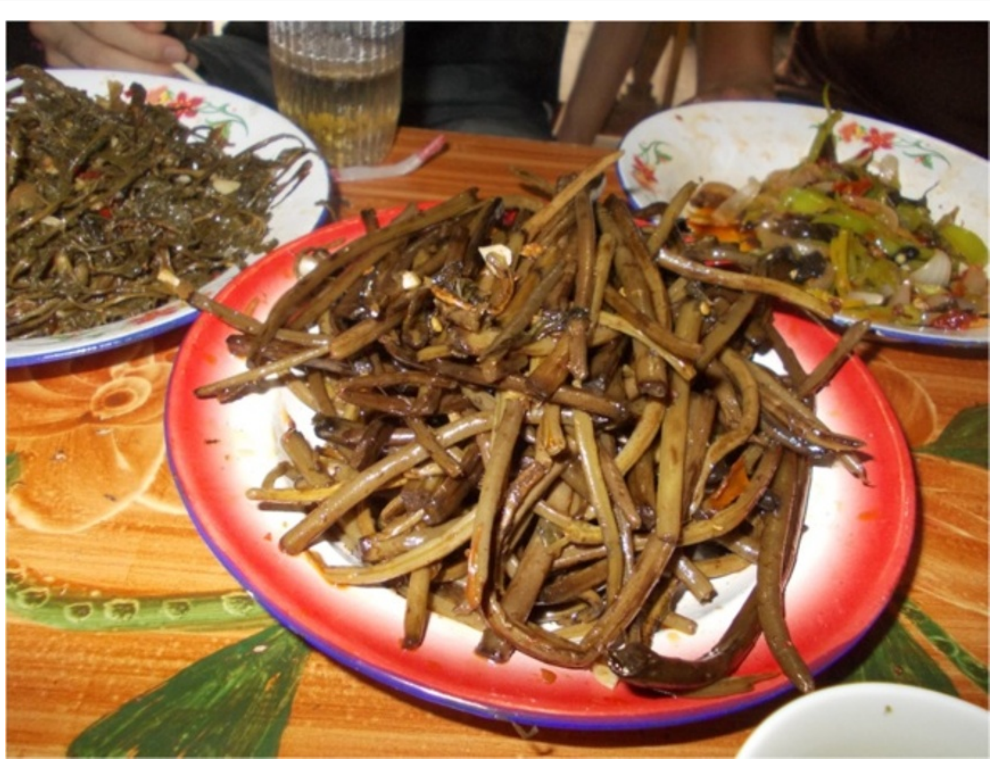

Figure 8 Lacto-fermented stalks of bracken fronds, served fried with chilli and pieces of bacon. 


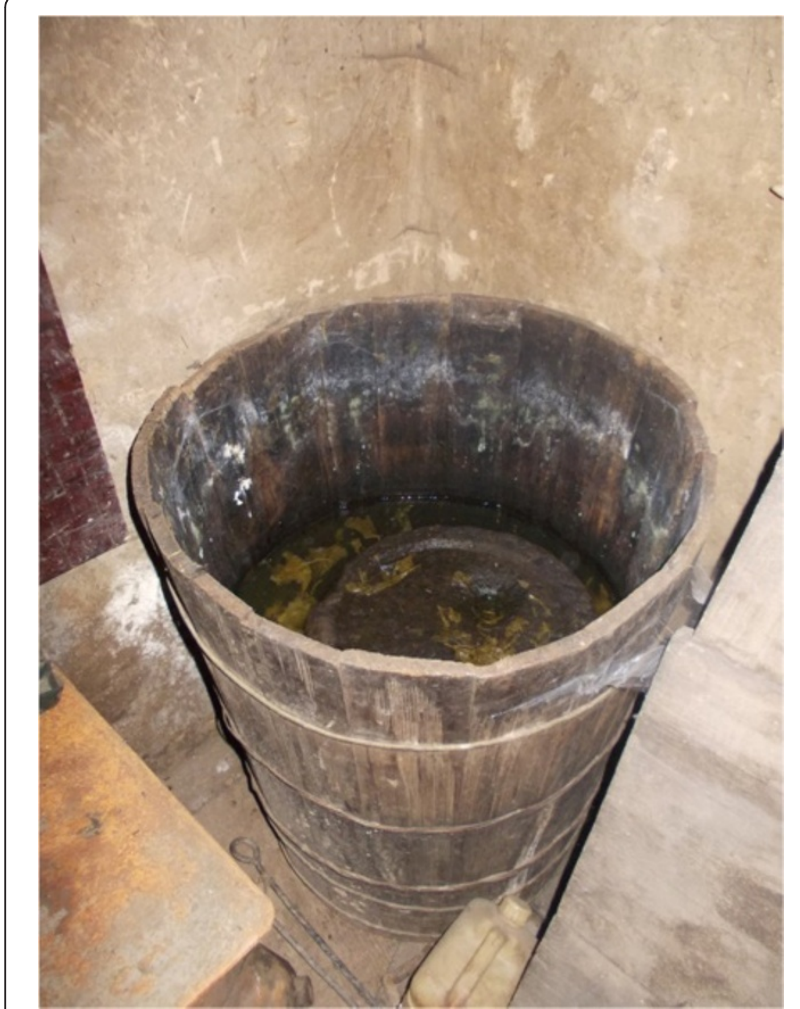

Figure 9 A barrel with lacto-fermented shoots of Helwingia japonica.

commonest plants in the roadsides) were widely used as emergency food and they are well remembered, although no one eats them nowadays.

Practically all families dry wild vegetables for further use, and many of them lacto-ferment wild vegetables in wooden barrels (around half of the families) while some have recently started freezing wild vegetables for winters in electric refrigerators (Figures 8, 9 and 10).

The most widely used wild vegetables are: Eleuterococcus spp., ferns - called collectively zhala (pinyin pronunciation), Helwingia japonica, Aralia chinensis, Allium victorialis, Pteridium aquilinum, Ixeris chinensis, Thlaspi arvense and Chenopodium album (Table 1). The first four of these taxa (Eleuterococcus spp., ferns, Helwingia japonica and Aralia chinensis) are also traded throughout the county and are mentioned even in the Zhouqu county annals as important sources of income for the locals [29].

It is interesting that some wild greens, which are very common species in the valley and are eaten in other parts of China, e.g. wild amaranths Amaranthus spp. and nettles Urtica spp. [6,26,27], are almost entirely ignored, regarded as inedible by most of the population, and eaten by very few individuals.

The most commonly eaten fruits are: wild pears Pyrus xerophila, wild plums Prunus salicina, Berchemia sinica, blackberries Rubus spp. (black fruited) and Eleagnus umbellata. The large number species of fruits reported in this study, compared to the central part of the Qinling Mts. [26,27], probably stems from the different landscape. Here, there is an abundance of natural hedgerows growing on steep banks dividing the field terraces, providing large masses of edible fruits near homesteads.

The number of wild taxa eaten in the studied valley is relatively large compared to most studies around the world. However, compared to the northern slope of the Qinling, the list is shorter, in spite of the similar methodology applied and similar research effort involved

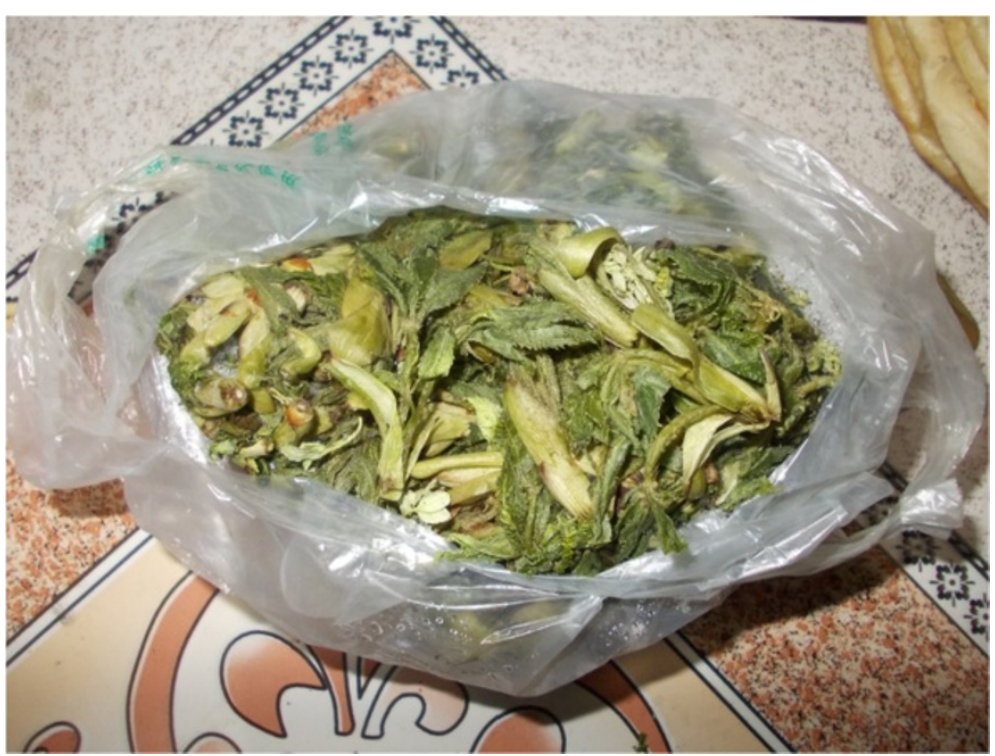

Figure 10 Freezing has recently become an alternative to lacto-fermenting or drying wild vegetables: frozen Eleuterococcus leaves. 
$[26,27]$. This may stem from the combined influences of two factors:

1. the local flora is relatively poorer here (the rainfall is lower, forest understory is poorer and the forest vegetation is more transformed by humans)

2. the local population is less interested in using all the potentially edible wild vegetables, for example the extremely abundant Amaranthus and Urtica are not utilized by the majority of the population.

In our previous papers [26,27] from northern Qinling we noted that local people seem to value forest wild greens over the ruderal taxa. This is even more visible in this part of the Qinling, where the gathering efforts are nearly exclusively oriented towards forest greens. Local people venture six $\mathrm{km}$ hikes up steep slopes in order to collect ferns, marsh marigolds Caltha palustris and bittercress Cardamine macrophylla, and do not bother to collect annual wild greens growing around their homesteads!

The culinary use of Caltha palustris is very interesting. In its raw state marsh marigold is a highly toxic plant, due to the presence of protoanemonin, and has not been eaten recently. We only have historical reports about its use in northern Europe, mainly from Estonia [39], and from the United States (as potherb - [40], or flower buds as caper substitutes - [41]). Some plant dictionaries have also recorded the edibility of Caltha leaves and roots, e.g. Kluk's Dykcyonarz Roślinny from eighteenth century Poland [42]. Protoanemonin is broken down by drying and long cooking $[43,44]$. In the study area, the plant is either dried and then used like other wild greens, or it is lacto-fermented after the initial blanching. The dried specimens of marsh marigolds we ate had no bitterness even prior to cooking. Further studies are needed to establish if the local population of marsh marigolds is a low-protoanemonin form easier to prepare as food than the European Caltha. For example, one of the authors' ( $Ł$ ) unpublished experiments show that $C$. palustris from Poland needs one to two hours of cooking to get rid of the bitterness caused by protoanemonin. Some species of the related genus Ranunculus have been eaten in Europe after only short cooking, as they are not as bitter as European Caltha, e.g. $R$. repens L. in Belarus and Poland [45], $R$. neapolitanus Ten. in Croatia [46], Ranunculus muricatus L. in Herzegovina [46] and R. ficaria L. in Slovakia [47], Ukraine [48], Romania and Hungary [49] and Italy [50].

It is interesting that the list of wild edible fruits is slightly longer than that of wild greens, as the reverse was usually the case in wild food studies in East Asia. The structure of the edible species list (similar number of wild greens and fruits) is very similar to that recorded among the Tibetans in Shangri-La [12] where 80 wild vegetable species and 78 edible fruit species were recorded. It is also more reminiscent of other regions of the world such as Africa $[51,52]$ and India $[53,54]$, where wild greens are used widely but not as many species as in the Qinling Mountains and other regions of East Asia, where the local biodiversity enables a choice from many species of wild vegetables.

Similarly to the Shaanxi part of the Qinling mountains, the number of fungi taxa collected is low. There were only four taxa mentioned by more than four informants, whereas as many as 153 species of edible mushrooms were identified in the area [34]. The two most commonly collected taxa (Grifola and Morchella) do not have original Tibetan names, but names borrowed from Chinese, which suggests that it was the Chinese who introduced their use. In contrast to the mushrooms, there is high consensus on wild vegetable and fruit names and all of them possess local Tibetan names.

The high level of bilinguality as far as plant names are concerned is worth emphasizing. Although bi- and trilinguality is very common in cultural edge areas (Zhouqu county has been a Tibetan-Chinese ethnic borderland for centuries), a nearly full bilinguality concerning plant names is a much rarer phenomenon. When we asked our informants why they know nearly all the local Chinese names of edible plants (although they speak only Tibetan in their villages) they explained that they need them when they talk about plants with people from the neighbouring Han villages. This is a proof that fortunately wild edible plants still constitute an important topic of conversation for the villagers and they maintain a high cultural status.

\section{Conclusions}

The number of wild taxa eaten in the studied valley is relatively large compared to most studies of edible plants from throughout the world. However, compared to the northern slope of the Qinling, the list is shorter, in spite of the similar methodology applied and similar research effort involved. The culinary use of the toxic Caltha palustris is worth emphasizing.

\section{Competing interests}

The authors state that they have no competing interests.

\section{Authors' contributions}

YK organized the expedition, led the interviews, identified most taxa and drafted parts of the text. $Ł$ wrote the first version of the article and processed the data. YK, ŁŁ, JK, FW, JH took active part in the interviewing process in the field, voucher specimen preparation and data processing, and read the final version of the manuscript. QG took part in the interviewing process, organized group meetings and helped to design the study. All authors read and approved the final manuscript.

\section{Acknowledgments}

Many thanks to the inhabitants of the studied villages for their willingness in sharing information on the use of the species. The program was

financially supported by the Forestry Research Foundation for the Public Service Industry of China $(2009,04004)$ and by the University of Rzeszów (Institute of Biotechnology and Basic Sciences, as well as a special grant from the rector of the university). Many thanks to prof. Xiaosi Guo for the identification of ferns. 


\section{Author details}

'College of Forestry, Northwest A\&F University, 712100 Yangling, Shaanxi, PR China. ${ }^{2}$ Department of Botany and Biotechnology of Economic Plants, Institute of Applied Biotechnology and Basic Sciences, University of Rzeszów, Werynia 502, 36-100 Kolbuszowa, Poland. ${ }^{3}$ Forestry Academy of Bailongjiang Forestry Administration Bureau, 746010 Liangshui, Gansu, PR China.

Received: 23 December 2013 Accepted: 27 January 2014

Published: 6 February 2014

\section{References}

1. Maurizio A: Die Geschichte unserer Pflanzennahrung: von den Urzeiten bis zur Gegenwart. Berlin: Parey; 1927.

2. Johns T: With bitter herbs they shall eat it: chemical ecology and the origins of human diet and medicine. Tucson: University of Arizona Press; 1990.

3. Bharucha Z, Pretty J: The roles and values of wild foods in agricultural systems. Phil Trans R Soc B 2010, 365:2913-2926.

4. Delang C: Not just minor forest products: the economic rationale for the consumption of wild food plants by subsistence farmers. Ecol Econ 2006 59:64-73.

5. Turner NJ, Łuczaj ŁJ, Migliorini P, Pieroni A, Dreon AL, Sacchetti LE, Paoletti MG: Edible and tended wild plants, traditional ecological knowledge and agroecology. Cr Rev Plant Sci 2011, 30(1-2):198-225.

6. Hu SY: Food Plants of China. Hongkong: The Chinese University Press; 2005.

7. Long C-L, Li H, Ouyang Z, Yang X, Li Q, Trangmar B: Strategies for agrobiodiversity conservation and promotion: a case from Yunnan China. Biodivers Conserv 2003, 12(6):1145-1156.

8. Xu Y, Tao G, Liu H, Yan K, Dao X: Wild vegetable resources and market survey in Xishuangbanna, Southwest China. Econ Bot 2004, 58(4):647-667.

9. Zhang XP, Wu JL, Li Y, Liu F, Wang JQ: Investigation on species resources and utilization of wild vegetable in Nabanhe watershed nature reserve, Xishuangbanna. J South Forestry Coll 2004, 24:21-24.

10. Ghorbani A, Langenberger G, Sauerborn J: A comparison of the wild food plant use knowledge of ethnic minorities in Naban River Watershed National Nature Reserve, Yunnan, SW China. J Ethnobiol Ethnomed 2012, $8: 17$.

11. Ghorbani A, Langenberger G, Liu J-X, Wehner S, Sauerborn J: Diversity of medicinal and food plants as non-timber forest products in Naban river Watershed National Nature Reserve (China): implications for livelihood improvement and biodiversity conservation. Econ Bot 2012, 66:178-191.

12. Ju Y, Zhuo J, Liu B, Long C: Eating from the wild: diversity of wild edible plants used by Tibetans in Shangri-la region, Yunnan, China. J Ethnobiol Ethnomed 2013, 9(1):28.

13. Khasbagan, Huai HY, Pei SJ: Wild plants in the diet of Arhorchin Mongol herdsmen in Inner Mongolia. Econ Bot 2000, 54(4):528-536.

14. Wujisguleng W, Khasbagen K: An integrated assessment of wild vegetable resources in Inner Mongolian Autonomous Region, China. J Ethnobiol Ethnomed 2010, 6:34

15. Weckerle CS, Huber FK, Yongping Y, Weibang S: Plant knowledge of the Shuhi in the Hengduan mountains, Southwest China. Econ Bot 2006, 60(1):3-23.

16. Huber FK, Ineichen $\mathrm{R}$, Yang K, Weckerle CS: Livelihood and conservation aspects of non-wood forest product collection in the Shaxi Valley, Southwest China. Econ Bot 2010, 64(3):189-204

17. Zou X, Huang F, Hao L, Zhao J, Mao H, Zhang J, Ren S: The socio-economic importance of wild vegetable resources and their conservation: a case study from China. Kew Bull 2010, 65:577-582.

18. Wang $L$, Wang $R$ : Research on the resources of wild vegetable plants in Tianshui of Gansu Province [甘肃天水野生蔬菜植物资源研究]. Forest By-Product and Speciality in China 2013, 122(1):76-77.

19. Hansen MH: Frontier people: Han settlers in minority areas of China. Vancouver: UBC Press; 2007.

20. Boesi A: Plant categories and types in Tibetan materia medica. Tibet $J$ 2005, 30(4/1):65

21. Boesi A: Plant knowledge among Tibetan populations. Memorie della Società Italiana di Scienze Naturali e del Museo Civico di Storia Naturale di Milano 2005, 33(1):33-48.

22. Liu Y, Dao Z, Yang C, Liu Y, Long C: Medicinal plants used by Tibetans in Shangri-la, Yunnan, China. J Ethnobiol Ethnomed 2009, 5:15.

23. DeLancey S: Lhasa Dialect. In Sino-Tibetan Languages (Vol. 3). Edited by Thurgood G, LaPolla RJ. London: Psychology Press; 2003:270-288.
24. Sun JT-S: Phonological profile of Zhongu: a new Tibetan dialect of Northern Sichuan. Lang Ling 2003, 4(4):769-836.

25. Tournadre N: Arguments against the Concept of 'Conjunct'/'Disjunct' in Tibetan. In Chomolangma, Demawend und Kasbek. Festschrift für Roland Bielmeier zu seinem 65. Geburtstag. Vol. 1. Edited by Huber B, Volkart M Widmer M, Schwieger P. ; 2008:281-308.

26. Kang Y, Łuczaj $Ł$, Yes $S$, Zhang S, Kang J: Wild food plants and wild edible fungi of Heihe valley (Qinling Mountains, Shaanxi, central China): herbophilia and indifference to fruits and mushrooms. Acta Soc Bot Pol 2012, 81(4):405-413.

27. Kang Y, Łuczaj $Ł$, Kang J, Zhang S: Wild food plants and wild edible fung in two valleys of the Qinling Mountains (Shaanxi, central China). J Ethnobiol Ethnomed 2013, 9(1):26.

28. Kang $Y$, Łuczaj $Ł$, Ye $S$ : The highly toxic Aconitum carmichaelii Debeaux as a root vegetable in the Qinling Mountains (Shaanxi, China). Genet Resour Crop Evol 2012, 59:1569-1575.

29. The Editorial Committee of Gansu Zhouqu Local Annals: Zhouqu County Annals. Beijing: Fangzhi Press; 2010 [甘肃舟曲县地方志编篡委员会, 舟曲 县志, 方志出版社: 北京,2010]

30. Croll EJ: Research methodologies appropriate to rapid appraisal: a Chinese experience. IDS Bull 1984, 15(1):51-56.

31. Long CL, Wang JR: Participatory rural appraisal: An introduction to principle, methodology and application. Kunming: Yunnan Science and Technology Press; 1996.

32. Sutrop U: List task and a cognitive salience index. Field Methods 2001, 3(13):263-276

33. Quinlan M: Considerations for collecting freelists in the field: examples from ethnobotany. Field Methods 2005, 17(3):219-234.

34. Guo Q, Gao Z, Song X, Li Y, Fu Z, Wang Y, He H, Tang Y: Macrofungi flora distribution and faunal study in Bailongjiang-Taohe forest region (白龙 江、洮河林区大型真菌资源及分布). J Northwest Forestry University 2010, 25(3):126-136

35. American Anthropological Association Code of Ethics. [http://www. aaanet.org/issues/policy-advocacy/upload/AAA-Ethics-Code-2009.pdf]

36. International Society of Ethnobiology Code of Ethics (with 2008 additions). [http://ethnobiology.net/code-of-ethics/]

37. Hammer O, Harper DAT, Ryan PD: PAST: Paleontological Statistics software package for education and data analysis. Paleontol Electron 2001, 4(1):9.

38. PAST version 2.17b. [http://folk.uio.no/ohammer/past]

39. Kalle R, Sõukand R: Historical ethnobotanical review of wild edible plants of Estonia (1770 s-1960s). Acta Soc Bot Pol 2012, 81(4):271-281.

40. Gray A: Manual of the Botany of the Northern United States. Boston and Cambridge: James Munroe \& Co.; 1848.

41. Porcher FP: Resources of the Southern Fields and Forests. Medical , Economical and Agricultural; being also a Medical Botany of the Southern States; with practical information on the useful properties of the trees, plants, and shrubs. Charleston: Walker, Evans and Cogswell; 1869.

42. Kluk K: Dykcyonarz roślinny. Tom I. Warszawa: Drukarnia Xięży Piarów; 1786.

43. Gmelin L: Fortsetzung des Handbuchs der Organischen Chemie. Part 4. Heidelberg: Carl Winter's Universitat-Buchhandlungs; 1866.

44. Berger A, Wachter H: Hunnius Pharmazeutisches Wörterbuch (in German). 8th edition. Berlin: Walter de Gruyter Verlag; 1998.

45. Łuczaj Ł, Köhler P, Pirożnikow E, Graniszewska M, Pieroni A, Gervasi T: Wild edible plants of Belarus: from Rostafiński's questionnaire of 1883 to the present. J Ethnobiol Ethnomed 2013, 9:21.

46. Łuczaj Ł, Zovko Končić M, Miličević T, Dolina K, Pandža M: Wild vegetable mixes sold in the markets of Dalmatia (Southern Croatia). J Ethnobiol Ethnomed 2013, 9:1.

47. Łuczaj Ł: Ethnobotanical review of wild edible plants of Slovakia. Acta Soc Bot Pol 2012, 81(4):245-255.

48. Łuczaj Ł: Brzozowy sok, "czeremsza" i zielony barszcz - ankieta etnobotaniczna wśród botaników ukraińskich. Birch sap, ramsons and green borsch - an ethnobotanical survey among Ukrainian botanists. Etnobiologia Polska 2012, 2:15-22.

49. Dénes A, Papp N, Babai D, Czúcz B, Molnár Z: Wild plants used for food by Hungarian ethnic groups living in the Carpathian Basin. Acta Soc Bot Pol 2012, 81(4):381-396.

50. Pieroni A: Gathered wild food plants in the upper valley of the Serchio River (Garfagnana), Central Italy. Econ Bot 1999, 53(3):327-341.

51. Addis G, Urga K, Dikasso D: Ethnobotanical study of edible wild plants in some selected districts of Ethiopia. Hum Ecol 2005, 33(1):83-118. 
52. Johns T, Kokwaro JO: Food plants of the Luo of Siaya district, Kenya. Econ Bot 1991, 45(1):103-113.

53. Sundriyal M, Sundriyal RC: Wild edible plants of the Sikkim Himalaya: marketing, value addition and implications for management. Econ Bot 2004, 58(2):300-315.

54. Rajasab AH, Isaq M: Documentation of folk knowledge on edible wild plants of north Karnataka. Indian J Tradit Know 2004, 3(4):419-429.

doi:10.1186/1746-4269-10-20

Cite this article as: Kang et al.: Wild food plants used by the Tibetans of Gongba Valley (Zhouqu county, Gansu, China). Journal of Ethnobiology and Ethnomedicine 2014 10:20.

\section{Submit your next manuscript to BioMed Central and take full advantage of:}

- Convenient online submission

- Thorough peer review

- No space constraints or color figure charges

- Immediate publication on acceptance

- Inclusion in PubMed, CAS, Scopus and Google Scholar

- Research which is freely available for redistribution 